\title{
DNA Binding
}

National Cancer Institute

\section{Source}

National Cancer Institute. DNA Binding. NCI Thesaurus. Code C18597.

Used in reference to proteins or low molecular weight solutes (ligands) that interact specifically with DNA, in either a DNA-sequence dependent or independent fashion. 